\title{
Acute Intermittent Porphyria in a Child with Severe Neuropathy
}

Elena Di Pierro ${ }^{1 \#}$, Francesca Granata ${ }^{1 \#}$, Cristiano Rosafio ${ }^{2 \#}$, Stefano Marchini ${ }^{3}$, Azzurra Guerra $^{2}$, Valentina Brancaleoni ${ }^{1}$, Lorenzo lughetti ${ }^{2}$ and Paolo Ventura ${ }^{3 *}$

${ }^{1}$ UOC Internal Medicine, IRCCS Ca Granda Foundation, Maggiore Policlinico Hospital, Milan, Italy

${ }^{2}$ Division of Paediatrics, Department of Medical and Surgical Sciences for Children and Adults, University of Modena and Reggio Emilia, Policlinico Hospital, Modena, Italy ${ }^{3}$ Division of Internal Medicine II-Centre for Porphyrias, Department of Medical and Surgical Sciences for Children and Adults, University of Modena and Reggio Emilia,

Policlinico Hospital, Modena, Italy

"Authors contributed equally to this paper

\begin{abstract}
Clinical presentation of acute intermittent porphyria before puberty is unusual. We diagnosed the non-erythroid variant form of this disease in a male child, who first presented, at the age of 6 years, with unexplained neurological symptoms and behavioural abnormalities. We also report the successful treatment, and the long-term clinical management.
\end{abstract}

Keywords: Acute porphyrias; Acute intermittent porphyria; Peripheral neuropathy; Heme; Heme arginate; Children

Abbreviations: AIP: Acute Intermittent Porphyria; ALA: DeltaAminolevulinic Acid; ANCA: Anti-Neutrophil Cytoplasmic Antibodies; APA: Acute Porphyric Attack; CPOX: Coproporphyrinogen Oxidase; HHV: Human Herpes Virus; HMBS: Hydroxymethyl-Bilane Synthase; PBG: Porphobilinogen; PBGD: Porphobilinogen Deaminase; PPOX: Protoporphyrinogen Oxidase

\section{Patient Presentation}

A 6-year-old boy of Moroccan origin, presented with severe and progressive weakness in the upper and lower limbs. The patient had also experienced progressive behavioural changes (irritable and oppositive) and calorie restriction over a 2-year period prior to admission. The patient's vital signs and his thorax and abdominal examinations were normal; however, he appeared poorly nourished (body-mass index $=14$ ). The patient was unable to manipulate objects, with bilateral hands, he had foot drop and showed a severe steppage gait. At neurological examination, the cranial nerve function tests resulted normal, while motor strength tests reduced (Table 1). Temperature, vibration, proprioception, light touch and pinprick sensations were also normal. No abnormalities were observed at magnetic resonance imaging of the brain and spinal cord, electrocardiogram, chest X-ray and abdomen $\mathrm{X}$-ray and ultrasound imaging. To test for Guillain-Barre Syndrome, a lumbar puncture together with a study of nerve conduction was performed. The cerebrospinal fluid analysis showed a glucose level of $47 \mathrm{mg} / \mathrm{dL}$ (reference values $45-80 \mathrm{mg} / \mathrm{dL}$ ) and a protein level of $20 \mathrm{mg} /$ $\mathrm{dL}(12-60 \mathrm{mg} / \mathrm{dL})$ without positivity for cell or Gram's staining; protein isoelectric focusing and immunoblotting assays showed a nonspecific "mirror pattern". Electromyography was suggestive for chronic axonal motor neuropathy. The peroneal and median nerves responses were bilaterally not recordable. White and red blood counts, serum levels of folate and vitamin B12 and basal hormone levels (sexual hormones and thyroid, hypophysis, adrenal gland-related hormones) were normal. In Table 1 the results for heavy metals assessment, transaminases and lipid status are reported. Tests were negative for infectious diseases (human immunodeficiency virus, hepatitis $\mathrm{C}$ virus, cytomegalovirus, EpsteineBarr virus, herpes simplex virus 1 and 2, human herpes virus (HHV) 6-8, Enterovirus and Borrelia Burgdorferi spp., tuberculosis (quantiFERONTB Gold In-Tube)) and for antinuclear antibodies (ANA), perinuclear anti-neutrophil cytoplasmic antibodies (ANCA), cytoplasmic ANCA, anti-gangliosides antibodies, and paraproteinemias. Screening for metabolic disorders characterized by neuropathic involvement showed no significant alteration with regard to acyl-carnitine profile, urinary organic acids levels and amino acids serum profile. The urine analysis surprisingly revealed a high level of total porphyrins, 3479 $\mathrm{g} / \mathrm{g}$ creatinine (ref.<110 g/g creatinine); delta-aminolaevulinic acid (U-ALA) $47.1 \mathrm{~mol} / \mathrm{mol}$ creatinine (ref. $<5 \mathrm{~mol} / \mathrm{mol}$ creatinine); and porphobilinogen (U-PBG) $82.4 \mathrm{~mol} / \mathrm{mol}$ creatinine (ref. $<1.5 \mathrm{~mol} /$ mol creatinine) (measured by Chromatography methods). These data strongly suggested the diagnosis of an acute porphyria.

Genetic analysis, obtained after informed consent from the patient and his family, identified a novel splice-site mutation in $H M B S$ gene, confirming the diagnosis of heterozygosity for AIP. The value of the PBGD activity in the erythrocytes was normal thus the ubiquitous promoter region including the exon 1 of the $H M B S$ gene (HMBS RefSeq NC_000011.10 119084871-119093549), was PCRamplified using primers and conditions as previously reported [1]. An $A>G$ substitution in the exon 1 donor splice site of $H M B S$ gene was identified (c.33+4 $A>G)$ confirming that the patient was affected by the non-erythroid variant form of AIP. Family studies also revealed that his asymptomatic mother and brother were equally heterozygous for the splice-site mutation, never described before. In order to explain the severity of the phenotype of the patient, the entire coding regions of $H M B S$ and of the genes of other acute porphyria such as delta aminolevulinic acid dehydratase (ALAD), coproporhyrinogen oxydase (CPOX), protoporhyrinogen oxidase (PPOX) and aminolevulinic acid synthase (ALAS1) were sequenced. In order to exclude long rearrangements, the multiplex ligation-dependent probe amplification was also performed as previously described [2], but no additional abnormalities were identified. However, family segregation analysis of common polymorphisms of $H M B S$ gene showed that the proband and the asymptomatic brother inherited two different paternal alleles

*Corresponding author: Ventura P, Department of Medical and Surgical Sciences for Children and Adults, University of Modena and Reggio Emilia, Policlinico Hospital, Modena, Largo del Pozzo 7141124 Modena, Italy, Tel: 059-4225542; Fax: 059-4224363; E-mail: paoloven@unimore.it

Received December 21, 2017; Accepted December 23, 2017; Published December 30, 2017

Citation: Pierro ED, Granata F, Rosafio C, Marchini S, Guerra A, et al. (2017) Acute Intermittent Porphyria in a Child with Severe Neuropathy. J Blood Lymph 8: 195. doi:10.4172/2165-7831.1000195

Copyright: $\odot 2017$ Pierro ED, et al. This is an open-access article distributed unde the terms of the Creative Commons Attribution License, which permits unrestricted use, distribution, and reproduction in any medium, provided the original author and source are credited. 


\begin{tabular}{|c|c|}
\hline & Value (reference range) \\
\hline Height & $113 \mathrm{~cm}\left(54^{\circ}\right.$ percentile $)$ \\
\hline Weight & $18 \mathrm{~kg}\left(25^{\circ}\right.$ percentile) \\
\hline Body-mass index & $14\left(10^{\circ}\right.$ percentile) \\
\hline Motor strength \\
\hline Upper portion of the arms \\
\hline Lower portion of the arms & $4 / 5$ \\
\hline Fingers & $2-3 / 5$ \\
\hline Upper legs & $0 / 5$ \\
\hline Lower legs & $4+/ 5$ \\
\hline Complete laboratory testing & $0-2 / 5$ \\
\hline Total cholesterol & \\
\hline High-density lipoprotein & $275 \mathrm{mg} / \mathrm{dL}(126-91 \mathrm{mg} / \mathrm{dL})$ \\
\hline Alanine transaminase & $99 \mathrm{mg} / \mathrm{dL}(>39 \mathrm{mg} / \mathrm{dL})$ \\
\hline Aspartate aminotransferase & $51 \mathrm{IU} / \mathrm{L}(8-20 \mathrm{IU} / \mathrm{L})$ \\
\hline Heavy metal levels & $53 \mathrm{IU} / \mathrm{L}(8-20 \mathrm{IU} / \mathrm{L})$ \\
\hline Urinary cadmium & \\
\hline Blood lead levels & $0.05 \mu \mathrm{\mu g} / \mathrm{L}(0-0.2 \mu \mathrm{\mu g} / \mathrm{L})$ \\
\hline
\end{tabular}

*Standard routine analysis, IU=international units.

Table 1: Patient characteristics.

(Figure 1, section A). Assuming the existence of a second mutation in the $H M B S$ gene inherited from the father, a region of about $2 \mathrm{~Kb}$ of distal promoter was also sequenced, but no additional abnormalities were identified. Moreover, no difference in common polymorphisms was found between two brothers carrying mutation, also excluding the hypothesis of an additional hypomorphic allele. In addition, HMBS gene expression confirmed that the proband and the asymptomatic brother had the same amount of $H M B S$ mRNA.

Specific therapy by intravenous infusion of Haem arginate (Normosang, Orphan Europe, Paris, France) at a dose of $3 \mathrm{mg} / \mathrm{kg} /$ day by central catheter for 3 consecutive days was started. Following this infusion, the general clinical conditions of the child progressively improved, with reduction in neurological deficits and food refusal. On the basis of urinary ALA and PBG values, together with clinical incomplete remission, a maintenance scheme of one dose of Heme Arginate infusion for 2 consecutive days per week was initiated during the following months, with continuous monitoring of clinical and biochemical (U-ALA, U-PBG) parameters. T0 at the time of diagnosis U-total porphyrins $3479 \mathrm{~g} / \mathrm{g}$ creatinine U-PBG $82,4 \mathrm{~mol} / \mathrm{mol}$ creatinine U-ALA $47,1 \mathrm{~mol} / \mathrm{mol}$ creatinine. T1 at 1 month of therapy. before therapy U-total porphyrins $2406 \mathrm{~g} / \mathrm{g}$ creatinine U-PBG $124 \mathrm{~mol} / \mathrm{mol}$ creatinine U-ALA $46,1 \mathrm{~mol} / \mathrm{mol}$ creatinine; after therapy U-total porphyrins $574 \mathrm{~g} / \mathrm{g}$ creatinine U-PBG $24 \mathrm{~mol} / \mathrm{mol}$ creatinine U-ALA $8,2 \mathrm{~mol} / \mathrm{mol}$ creatinine. T2 at 2 month of therapy. before therapy U-total porphyrins $2687 \mathrm{~g} / \mathrm{g}$ creatinine U-PBG 73,9 $\mathrm{mol} / \mathrm{mol}$ creatinine U-ALA $19,2 \mathrm{~mol} / \mathrm{mol}$ creatinine after therapy U-total porphyrins 1893 $\mathrm{g} / \mathrm{g}$ creatinine U-PBG $22,2 \mathrm{~mol} / \mathrm{mol}$ creatinine U-ALA $2,8 \mathrm{~mol} / \mathrm{mol}$ creatinine. T3 at 3 month of therapy. before therapy U-total porphyrins $2068 \mathrm{~g} / \mathrm{g}$ creatinine U-PBG $88,7 \mathrm{~mol} / \mathrm{mol}$ creatinine U-ALA $53,5 \mathrm{~mol} /$ mol creatinine after therapy U-total porphyrins $1964 \mathrm{~g} / \mathrm{g}$ creatinine U-PBG $22,4 \mathrm{~mol} / \mathrm{mol}$ creatinine U-ALA $6,2 \mathrm{~mol} / \mathrm{mol}$ creatinine. T6 at 6 month of therapy. before therapy U-total porphyrins 2829 g/g creatinine U-PBG $93,7 \mathrm{~mol} / \mathrm{mol}$ creatinine U-ALA $34,9 \mathrm{~mol} /$ mol creatinine after therapy $\mathrm{U}$-total porphyrins $975 \mathrm{~g} / \mathrm{g}$ creatinine U-PBG 32,9 $\mathrm{mol} / \mathrm{mol}$ creatinine U-ALA $7 \mathrm{~mol} / \mathrm{mol}$ creatinine. T1 food refusal disappeared and improved strength in lower limbs. T2 almost normalized strength in lower limbs, improved strength in upper limbs almost normal behavior. T6 upper and lower limb neurological signs and gait anomalies were completely normalized (Figure 1, section B). After 6 months of therapy, upper and lower limb neurological signs and gait anomalies were completely normalized, together with significant improve in behaviour.

\section{Discussion}

Acute Intermittent Porphyria (AIP) is a low-penetrance autosomal dominant disorder of heme synthesis, characterized by a highly variable clinical presentation [3]. AIP is caused by mono-allelic mutations in the hydroxymethyl-bilane synthase gene (HMBS), coding for the third enzyme in the heme biosynthetic pathway. The mutation predisposes the carriers to sporadic life-threatening acute neurological attacks that require additional triggering factors [4]. Prevalent clinical features of an acute attack include severe addominal pain, variably accompanied by neurological symptoms (mostly peripheral acute neuropathy, ofteninvolving proximal portion of the limbs) and psychiatric abnormalities (such as behaviour alterations, agitation, hallucinations). Acute attacks may also present with cardiovascular signs (tachycardia and/ or hypertension) or hyponatremia that can lead to seizures or even coma [5]. A higher incidence of seizures was reported in children who presented after treatment with porphyrinogenic anti-seizure medications [6]. Acute porphyria attacks are reported as usually occurring in second to third decades of life, very rarely in childhood and by far more common in females than males [7]. A recent revisiting of literature shows that although 70 paediatrics AIP cases have been reported, the diagnosis and the management of AIP in children has been and it is still challenging [8]. We report an unusual AIP phenotype in a 6 years old child of Moroccan origin.

An acute exacerbation of AIP is diagnosed on the basis of increased urinary levels of aminolevulinic acid (ALA) and porphobilinogen (PBG) [9]. However, if specific tests are conducted delayed with respect to clinical manifestations, the measurements are inconclusive; thus a diagnosis of AIP may be based solely on reduced erythrocyte HMBS activity. The latter can be however normal in cases affected by the nonerythroid variant form of AIP (about $10-15 \%$ of all AIP patients). This condition is due to mutations affecting a specific region of the HBMS gene, thereby only affecting HBMS activity in non-erythroid cells [10]. Our patient presented with high urinary porphyrin (uroporphyrins), ALA and PBG levels and normal erythroid HBMS activity suggesting a diagnosis of non-erythroid variant of AIP. The majority of mutations causing this form of AIP are exon 1 splicing defects; seven different substitutions from +1 to +5 position in the canonical splicing donor site have been reported. All these mutations result in the activation of a cryptic splicing site $67 \mathrm{bp}$ downstream in intron 1, leading to a frame shift and causing a premature stop codon in exon $4[11,12]$. It is possible that the $c .33+4 \mathrm{~A}>\mathrm{G}$ mutation described herein could have the same effect on HMBS mRNA, even if the abnormal isoform was not found during qualitative mRNA analysis.

Severe neurological involvement characterized by ataxia with progressive neurological deterioration has also been described, but mostly in rare cases of children with homozygous dominant acute porphyrias (mostly AIP) [7]. No other mutation in the coding sequence of the gene was expected in our patient given the normal HMBS activity in the erythrocytes. The absence of other mutations in distal promoter region of $H M B S$ and in the coding regions of $A L A S 1, A L A D$, $C P O X$ and $P P O X$ genes did not allow us to full clarify the severity of the phenotype, yet. However, other paediatric cases with severe phenotype of AIP are reported carrying only one mutation in the HMBS gene [8].

If a second mutation should exist, it should be located in the non- 


\section{(Section A)}

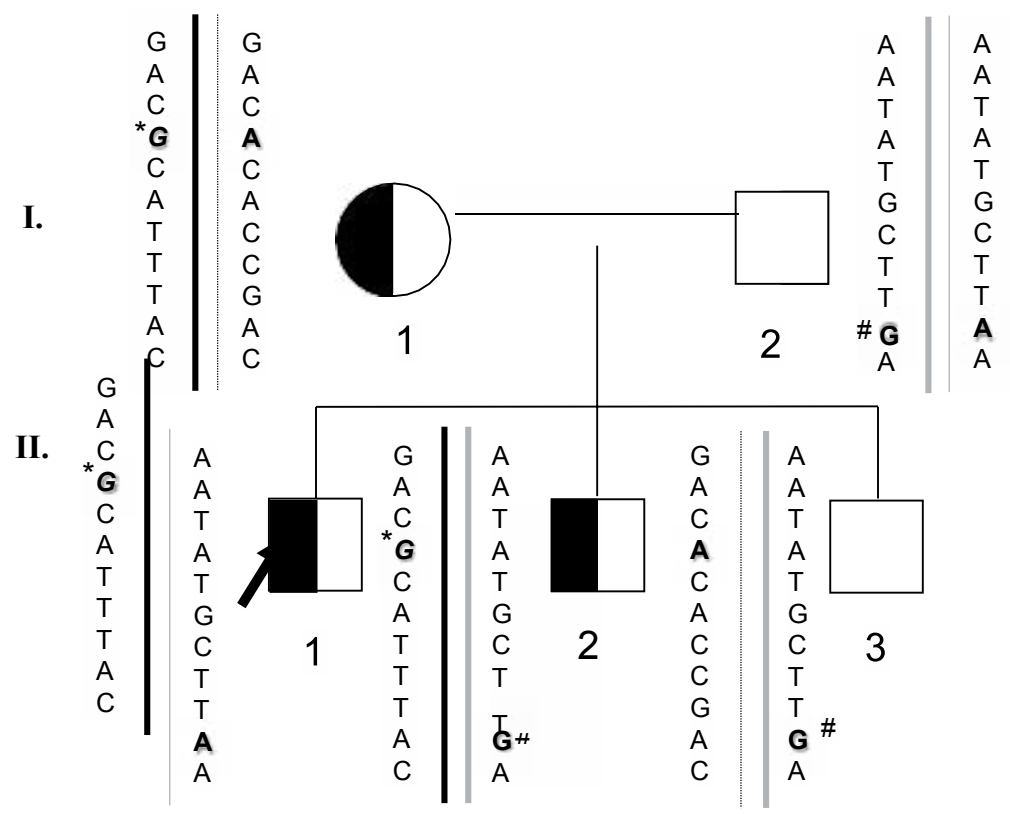

(Section B)

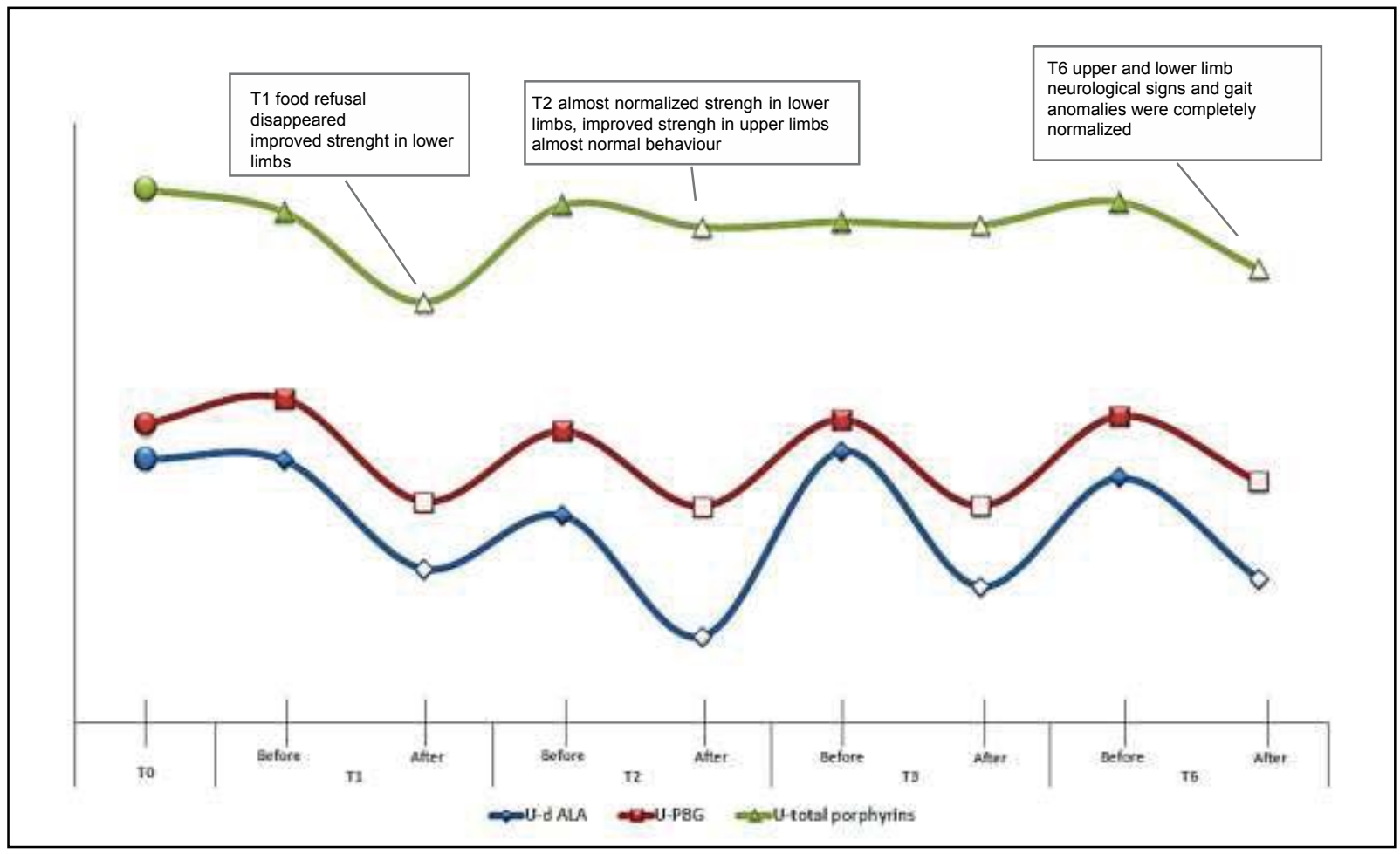

Figure 1

Section A: Genetic assessment of $H M B S$ gene and family segregation studied. The arrow points to the patient described in this case report. The carriers of c.33+4 $A>G$ mutation are depicted in black. The star indicates the mutated allele while the hashtag the difference between the two paternal alleles.

Section B: Improvement in patient symptoms [urine aminolevulinic acid (ALA), porphobilinogen (PBG), and total porphyrin levels; food refusal; motor strength; and neurological signs] throughout treatment. 
analysed intron 1 of $H M B S$ gene. The mutation should be carried by the paternal allele, which is the only difference between asymptomatic and symptomatic brothers carrying mutation. Anyway, the equal amount of $H M B S$ mRNA found in the proband and in the asymptomatic brother excludes the hypothesis that an additional hypomorphic allele could be inherited. We can suppose that other non-pophyria related genes perhaps located on the same chromosome of $H M B S$ gene (chr11) could modulate the clinical phenotype of AIP without however altering $H M B S$ gene expression.

Specific treatment for acute attack involves identifying and resolving any possible triggering factor, including stress, inadequate nutritional intake, infections and drugs or chemicals known to exacerbate porphyria. The mainstay of acute attack therapy is haem infusion [as hematin (Panhematin ${ }^{\otimes}$ ) or Heme arginate (Normosang $\left.{ }^{\circledR}\right)$ at the dosage of $2-4 \mathrm{mg} / \mathrm{kg}$ intravenously once/day for 3-4 days. Heme acts through a negative feedback on heme pathway, resulting in fast reduction in ALA and PBG accumulation: it resolves most of acute crisis in 2-4 days. Whilst glucose also down-regulates the heme pathway and may be helpful in resolving acute porphyria attacks $[13,14]$.

No literature for heme-arginate therapy in children for symptomatic AIP was available. Because of the elevated levels of ALA and PBG and the severe neurological impairment, heme-based therapy was initiated as described (by infusion through a central venous catheter) but it was prolonged to twice-a-week infusions following unsatisfactory outcomes during first-line therapy (Figure 1, section B). A gradual improvement in the patient's psycho-neurologic involvement resulted, with no acute therapy-related complications. Urinary levels of ALA and PBG dramatically decreased following each infusion but failed to reach reference intervals. AIP diagnosis in our patient was confirmed by DNA analysis, which also excluded other possible abnormalities in other genes of heme synthetic pathway [15]. The dissociation between clinical and biochemical outcome emphasizes uncertainties in pathological issues of AIP. The high urinary porphyrin, ALA and PBG levels, even outside of clinical exacerbations gives rise to the problem of a maintenance therapy.

Additional issues concerning the long-term clinical management of the patient include: how long maintenance therapy should be continued; the possibility of other treatment options; the risk of infections or other complications (thrombosis) of the central venous line; the possibility of iron overload; the effects of this experience on the psychological development of the patient.

In adult patients affected by severe forms of acute porphyrias liver transplantation has been considered an option leading to permanent cure of all acute porphyrias [16]. No literature for liver transplantation in children for symptomatic AIP is currently available. Recent experimental trials of gene-based therapy offer new promising opportunities for the treatment of acute porphyrias, even in long-term management [17].

Diagnosis of acute porphyrias is made less often than their prevalence justifies. Many patients remain undiagnosed, especially when presenting with unusual phenotypes [18]. An awareness of their possible multiform clinical presentations is imperative for successful diagnosis and treatment in order to prevent the escalation of symptoms, which may result in lethal consequences. Also paediatricians and neurologist should be made aware of insidious clinical patterns of acute porphyrias in children, presenting with, but not only, unexplained neurological symptoms.

\section{Acknowledgements}

We wish to thank P. Bergonzini, MD. C. Fusco, MD and the whole residents, attendants, interns and nursery staff of the Pediatrics Unit of Policlinico Hospital of Modena for their incomparable collaboration in the clinical management of this complex case. Native English and technical editing and styling prior to submission was performed by Melanie Gatt, on behalf of Springer Healthcare Communications. The editorial assistance was supported by funding from Orphan Europe.

\section{Funding Source}

This work was supported in part by grants from the Italian Ministry of Health (GR-2011-02347129) and from Fondazione IRCCS Ca'Granda Ospedale Maggiore Policlinico.

\section{Financial Disclosure}

The authors have no financial relationships relevant to this article to disclose.

\section{Conflict of Interest}

Paolo Ventura has been involved as consultant in advisory boards and has received funding for consultation, research and lecturing from Orphan Europe Italy. There are no other competing interests to declare for him and for all the other coauthors.

\section{Compliance with Ethics Guidelines}

All procedures followed were in accordance with the ethical standards of the responsible committee and with the Helsinki Declaration of 1975 , as revised in 2000: informed consent to make the diagnostic tests and to publish the results in this report were obtained from the people included in this study.

\section{Contributors' Statements}

Dr. Rosafio and Dr. Ventura made the diagnosis, managed the treatment and the follow up and drafted the initial manuscript; $\mathrm{Dr}$. Guerra contributed to make diagnosis and to manage the treatment and the follow up; Dr. Marchini carried out the biochemical analyses; Dr. Granata and Dr. Brancaleoni carried out the genetic analyses; Dr. Di Pierro carried out the genetic analyses and reviewed and revised the manuscript; Prof. lughetti critically reviewed the manuscript. All authors approved the final manuscript as submitted and agree to be accountable for al aspects of the work. None of the authors received any form of payment for the production of this manuscript.

\section{References}

1. Brancaleoni V, Granata F, Colancecco A, Tavazzi D, Cappellini MD, et al (2012) Seven novel genetic mutations within the 5'UTR and the housekeeping promoter of HMBS gene responsible for the non-erythroid form of acute intermittent porphyria. Blood Cells, Molecules \& Diseases 49: 147-151.

2. Di Pierro E, Brancaleoni V, Besana V, Cappellini MD (2009) Multiplex ligationdependent probe amplification: a novel approach for genetic diagnosis of Porphyria. Journal of Human Genetics 54: 479-487.

3. Elder GH, Hift RJ, Meissner PN (1997) The acute porphyrias. Lancet 349: 1613-1617.

4. Puy H, Gouya L, Deybach JC (2010) Porphyrias. Lancet 375: 924-937.

5. Ventura P, Cappellini MD, Biolcati G, Guida CC, Rocchi E, et al. (2014) A challenging diagnosis for potential fatal diseases: recommendations for diagnosing acute porphyrias. European Journal of Internal Medicine 25: 497-505.

6. Birchfield RI, Cowger ML (1996) Acute intermittent porphyria with seizures Anticonvulsant medication-induced metabolic changes. American Journal of Diseases of Children 112: 561-565.

7. Elder GH (1997) Hepatic porphyrias in children. Journal of Inherited Metabolic Disease 20: 237-246.

8. Balwani M, Singh P, Seth A, Debnath EM, Naik H, et al. (2016) Acute Intermittent Porphyria in children: A case report and review of the literature. Molecular Genetics and Metabolism119: 295-299.

9. Sandberg S, Elder GH (2004) Diagnosing acute porphyrias. Clinical Chemistry 50: 803-805.

10. Sassa S (1996) Diagnosis and therapy of acute intermittent porphyria. Blood Reviews 10: 53-58.

11. Mustajoki S, Pihlaja H, Ahola H, Petersen NE, Mustajoki $P$, et al. (1998) Three 
Citation: Pierro ED, Granata F, Rosafio C, Marchini S, Guerra A, et al. (2017) Acute Intermittent Porphyria in a Child with Severe Neuropathy. J Blood Lymph 8: 195. doi:10.4172/2165-7831.1000195

Page 5 of 5

splicing defects, an insertion, and two missense mutations responsible for acute intermittent porphyria. Human Genetics 102: 541-548.

12. Puy H, Gross U, Deybach JC, Robreau AM, Frank M, et al. (1998) Exon 1 donor splice site mutations in the porphobilinogen deaminase gene in the nonerythroid variant form of acute intermittent porphyria. Human Genetics 103: 570-575

13. Elder GH, Hift RJ (2001) Treatment of acute porphyria. Hosp Med 62: 422-425.

14. Stein P, Badminton M, Barth J, Rees D, Stewart MF, et al. (2013) Best practice guidelines on clinical management of acute attacks of porphyria and their complications. Ann Clin Biochem 50: 217-223.

15. Hessels J, Voortman G, van der Wagen A, van der Elzen C, Scheffer H, et al. (2004) Homozygous acute intermittent porphyria in a 7-year-old boy with massive excretions of porphyrins and porphyrin precursors. Journal of Inherited Metabolic Disease 27: 19-27.

16. Singal AK, Parker C, Bowden C, Thapar M, Liu L, et al. (2014) Liver transplantation in the management of porphyria. Hepatology 60: 1082-1089.

17. Yasuda M, Gan L, Chen B, Kadirvel S, Yu C, et al. (2014) RNAi-mediated silencing of hepatic Alas1 effectively prevents and treats the induced acute attacks in acute intermittent porphyria mice. Proceedings of the National Academy of Sciences of the United States of America111: 7777-7782.

18. Ventura P, Cappellini MD, Rocchi E (2009) The acute porphyrias: a diagnostic and therapeutic challenge in internal and emergency medicine. Internal and Emergency Medicine 4: 297-308. 